\title{
Microsurgical resection of a high-grade occipital arteriovenous malformation after staged embolization
}

\author{
Liyong Sun, MD, Hongqi Zhang, MD, and Jian Ren, MD
}

Department of Neurosurgery, Xuanwu Hospital, Capital Medical University, Beijing, China

The authors demonstrate the case of a 16-year-old girl with a large symptomatic occipital arteriovenous malformation (AVM). The staged embolization was performed to downgrade the AVM from Spetzler-Martin (S-M) Grade IV (Supplementary S-M Grade 7) to Grade III (Supplementary S-M Grade 5). The patient developed a subacute progressive visual field defect after the final time of embolization. MRI revealed an enlarged draining venous ectasia of the AVM compressing the visual cortex. Postoperatively, good radiological results were achieved, and the patient reported an improvement in her symptoms. The detailed operative technique and surgical nuances (including the surgical features of the AVM postembolization) of the marginal dissection and removal are illustrated in this video atlas.

The video can be found here: https://youtu.be/2MZq5patcJl.

KEYWORDS AVM; arteriovenous malformation; embolization; resection; posterior interhemispheric approach 